\title{
KỸ THUẬT TÁCH BA ĐỈNH CHẬP TRONG PHỔ BỨC XẠ GAMMÁ
}

\author{
Trịnh Ngọc Pháp ${ }^{a^{*},}$ Mai Xuân Trung ${ }^{b}$
}

\author{
${ }^{a}$ Trung tâm Thông tin Thu viện, Truờng Đại học Đà Lạt, Lâm Đồng, Việt Nam \\ ${ }^{b}$ Khoa Kỹ thuật Hạt nhân, Truoòng Đại học Đà Lạt, Lâm Đồng, Việt Nam \\ Lịch sử bài báo \\ Nhận ngày 08 tháng 10 năm 2015 | Chỉnh sửa ngày 20 tháng 04 năm 2016 \\ Chấp nhận đăng ngày 29 tháng 08 năm 2016
}

\begin{abstract}
Tóm tắt
Trong quá trình xủ̉ lý phổ bức xạ, vấn đề tách hai hay ba đỉnh chập trong phân tích đỉnh phổ năng luợng bưc xa Gamma là thuờng gặp. Kỹ thuật tách hai đỉnh chập đã được công bố trong các kết quả nghiên cúu của Mai và Võ (2015). Trong bài báo này chúng tôi trình bày kỹ thuật tách ba đỉnh chập bằng thuật toán Levenberg-Marquardt với nền phông tuyến tính hoạc phi tuyến. Thuật toán đã được áp dụng thành công trên các phổ bức xạ đo tù detector nhấp nháy hay bán dẫn.
\end{abstract}

Từ khóa: Tách đỉnh chập; Phổ Gamma; Thuật toán Levenberg-Marquardt.

\section{MỞ ĐẦU}

Trong quá trình ghi đo bức xạ ở các phòng thí nghiệm, vấn đề tách hai hay ba đỉnh chập thường xảy ra. Sự chủ động phân tích phổ trên các nền phông tuyến tính hoặc phi tuyến xuất phát từ phổ thực nghiệm là ý tưởng gợi mở để chúng tôi xây dựng chương trình phân tích phổ tách ba đỉnh chập trong việc xử lý các phổ Gamma đo trên các hệ phổ kế với detector nhấp nháy hoặc bán dẫn.

\section{CƠ SỞ LÝ THUYẾT}

\subsection{Các đỉnh chập trong phổ gamma}

Ba đỉnh chập trong phổ Gamma được chuẩn hóa dưới dạng hàm Gauss và theo biểu thức (1) (John, 2001).

$$
y(i)=A_{1} \exp \left(-\frac{\left(i-i_{1}\right)^{2}}{2 \sigma^{2}}\right)+A_{2} \exp \left(-\frac{\left(i-i_{2}\right)^{2}}{2 \sigma^{2}}\right)+A_{3} \exp \left(-\frac{\left(i-i_{3}\right)^{2}}{2 \sigma^{2}}\right)+B
$$

\footnotetext{
* Tác giả liên hệ: Email: phaptn@dlu.edu.vn
} 
Trong đó: $\mathrm{B}$ là hàm đường phông; $\mathrm{A}_{1}, \mathrm{~A}_{2}, \mathrm{~A}_{3}$ là giá trị biên độ của ba đỉnh phổ tương ứng; $\mathrm{i}$ là số kênh; $\mathrm{i}_{1}, \mathrm{i}_{2}, \mathrm{i}_{3}$ ứng với vị trí kênh của mỗi đỉnh; $\sigma$ là độ lệch chuẩn của đỉnh Gauss. Đường cong trên được mô tả bằng hàm mô hình như trong công thức (2).

$$
f\left(i, a_{1}, a_{2}, a_{3}, a_{4}, a_{5}, \mathrm{a}_{6}, \mathrm{a}_{7}\right)=a_{1} e^{-\frac{\left(i-a_{2}\right)^{2}}{2 a_{7}}}+a_{3} e^{-\frac{\left(i-a_{4}\right)^{2}}{2 a_{7}}}+a_{5} e^{-\frac{\left(i-a_{6}\right)^{2}}{2 a_{7}}}+B(i)
$$

Trong đó các tham số $a_{1}, a_{3}, a_{5}$ tương ứng là độ cao của đỉnh trên nền phông; $a_{2}$, $a_{4}, a_{6}$ tương ứng là vị trí kênh (năng lượng) của các đỉnh; $a_{7}$ là độ phân tán của đỉnh phổ liên hệ với độ rộng cực đại nửa chiều cao (John, 2001), $\mathrm{a}_{7}=\left(\frac{F W H M}{2.355}\right)^{2}=\left(\frac{\mathrm{w}}{2.355}\right)^{2}$ với FWHM = w là độ rộng cực đại nửa chiều cao đỉnh phổ; B(i) là dạng đường phông tuyến tính bậc nhất, bậc hai hoặc đường phông phi tuyến có dạng bậc thang. Một số dạng hàm mô tả phông bậc thang như trong công thức (3) (John, Michael, Christopher, \& William, 1990).

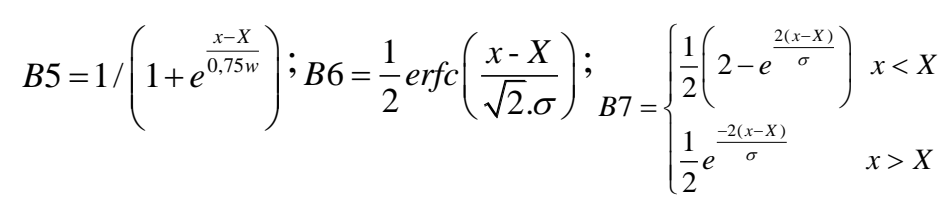

Trong đó: $x$ là số kênh; $X$ là vị trí tại tâm đỉnh; erfc là hàm bù sai số như trong (4).

$$
\operatorname{erfc}(x)=1-\operatorname{erf}(x) \text { với } \operatorname{erf}(x)=\frac{2}{\sqrt{\pi}} \int_{0}^{x} e^{-t^{2}} d t
$$

\subsection{Thuật toán Levenberg-Marquardt}

Phần này sẽ nêu thuật toán Levenberg-Marquardt (Hình 1) và các biểu thức của thuật toán. Vấn đề cần giải quyết của bài toán là cực tiểu hóa hàm đối tượng (Jorge, 1997) trên dáng điệu của nền phông đã lựa chọn cho phù hợp đối với phổ Gamma thực nghiệm, được biểu diễn như trong (5).

$$
\phi(x)=\frac{1}{2} \sum_{i=1}^{n}\left(y_{i}-F\left(i, a_{1}, a_{2}, a_{3}, a_{4}, a_{5}, a_{6}, a_{7}\right)\right)^{2}=\frac{1}{2} \sum_{i=1}^{n} r_{i}^{2}\left(a_{1}, a_{2}, a_{3}, a_{4}, a_{5}, a_{6}, a_{7}\right)
$$




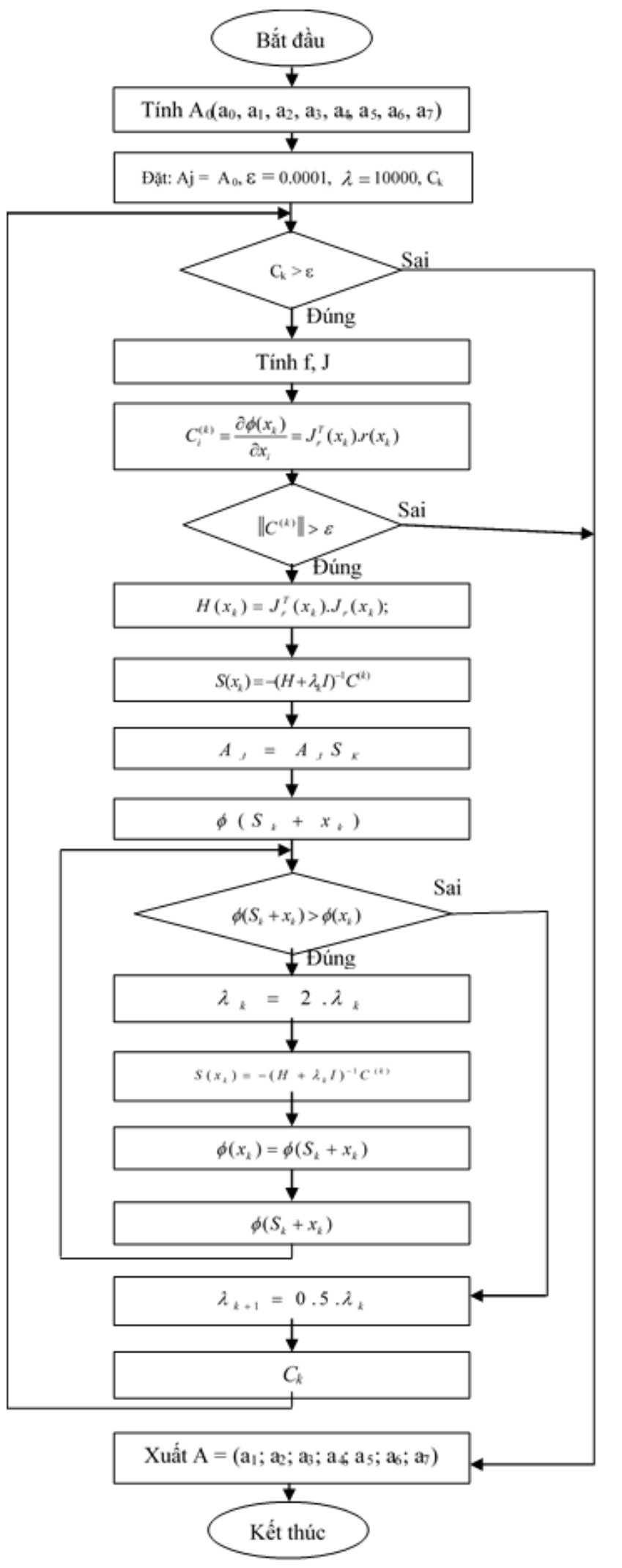

Hình 1. So đồ thuật toán tính các hệ số của đỉnh chập 
Trong đó $\mathrm{y}_{\mathrm{i}}$ là số đếm tương ứng với kênh $\mathrm{i} ; F\left(i, a_{1}, a_{2}, a_{3}, a_{4}, a_{5}, a_{6}, a_{7}\right)$ là hàm mô hình; $r_{i}$ là các thặng dư tương ứng với kênh trong vùng khảo sát.

Xây dựng thuật toán tách ba đỉnh chập chính là giải hệ phương trình (John, 2001; Jorge, 1997; David \& Yinyu, 2008) như trong (6).

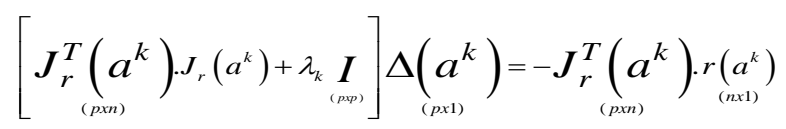

Trường hợp 3 đỉnh thì $\mathrm{p}=7$, tương ứng với 7 tham số cần tìm là $a_{1}, a_{2}, a_{3}, a_{4}, a_{5}$, $a_{6}, a_{7} ; J_{r}\left(a^{k}\right)$ là ma trận Jacobian của ma trận $r\left(a^{k}\right)=\left(r_{1}\left(a^{k}\right), r_{2}\left(a^{k}\right), \ldots ., r_{n}\left(a^{k}\right)\right)$ ở phép lặp thứ k; $J_{r}^{T}\left(a^{k}\right)$ là ma trận Jacobian chuyển vị; $\lambda_{k}$ là tham số được chọn theo thuật toán; $\mathrm{I}_{(\mathrm{pxp})}$ là ma trận đơn vị.

\subsection{Xác định diện tích đỉnh và sai số}

Diện tích đỉnh trong khoảng kênh từ $i-a, i+a$ được tính như trong (7).

$$
S(a)=\int_{i-a}^{i+a} A \exp \left(-\frac{\left(i-i_{0}\right)^{2}}{2 \sigma^{2}}\right) d i+\int_{i-a}^{i+a} B(i) d i
$$

Số đếm tại vị trí của các đỉnh sau khi tách có dạng:

$$
\mathrm{f}_{1}=a_{1} e^{-\frac{\left(x-a_{2}\right)^{2}}{2 a_{7}}}+B ; \mathrm{f}_{2}=a_{3} e^{-\frac{\left(x-a_{4}\right)^{2}}{2 a_{7}}}+B ; \mathrm{f}_{3}=a_{5} e^{-\frac{\left(x-a_{6}\right)^{2}}{2 a_{7}}}+B
$$

\section{CHƯƠNG TRÌNH TÍNH TOÁN}

Chương trình xử lý các đỉnh chập được viết bằng Matlab (Phạm, Ngô, Lê, \& Nguyễn, 2007; Nguyễn \& Nguyễn, 2006), với các chức năng bao gồm đọc và vẽ phổ năng lượng thu được từ thực nghiệm, chuẩn năng lượng theo kênh, chuẩn FWHM theo năng lượng, phân tích tách ba đỉnh năng lượng chồng chập trên các nền phông phi tuyến và tuyến tính, xác định diện tích của các đỉnh bị chập và sai số diện tích đỉnh. 


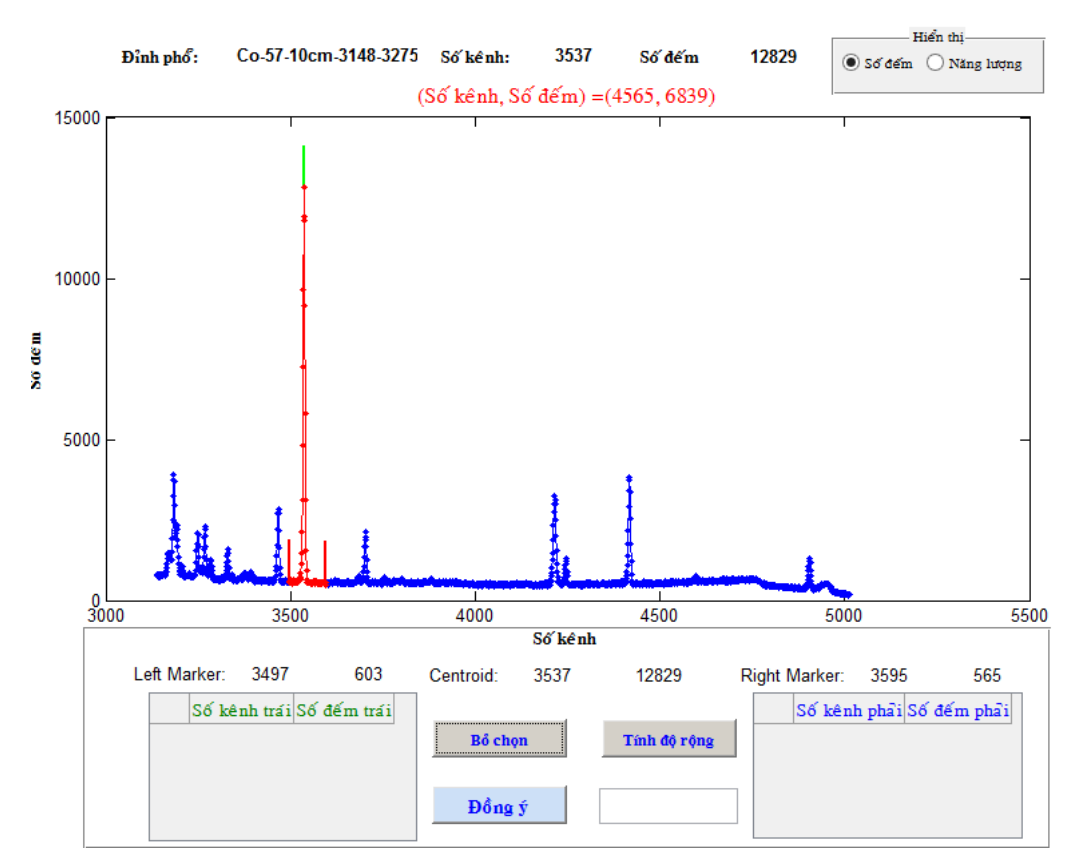

Hình 2. Giao diện tìm chọn vùng đỉnh

Một số chức năng chính trong phần mềm tách đỉnh chập:

- Chuẩn năng lương và độ rộng đỉnh FWHM: Chức năng này nhằm chuẩn tương quan năng lượng theo kênh. Từ đó dễ dàng xác định được năng lượng của tia Gamma phát ra, đồng thời cũng chuẩn độ rộng đỉnh theo năng lượng;

- Đọc và vẽ phổ: Chương trình đọc các số liệu thu được của phổ từ thực nghiệm bằng file excel (*.xls, *.xlsx), và được thiết lập mặc định đọc số liệu của tập tin có chứa số kênh, số đếm. Trong chương trình con này, ngoài việc chọn được vị trí kênh ta còn xác định được năng lượng tương ứng với vị trí kênh đã chọn. Từ các số liệu phổ thực nghiệm kết hợp với đường phông đã chọn sẽ vẽ được phổ trên nền phông;

- Tính toán hệ số a tù̀ thưc nghiệm: Chức năng của này là dựa trên các đỉnh trong vùng được chọn để chọn các hệ số ban đầu cho quá trình tách đỉnh. Các hệ số đó sẽ xác định được vị trí của từng đỉnh con sau khi tách và độ rộng của đỉnh chập; 


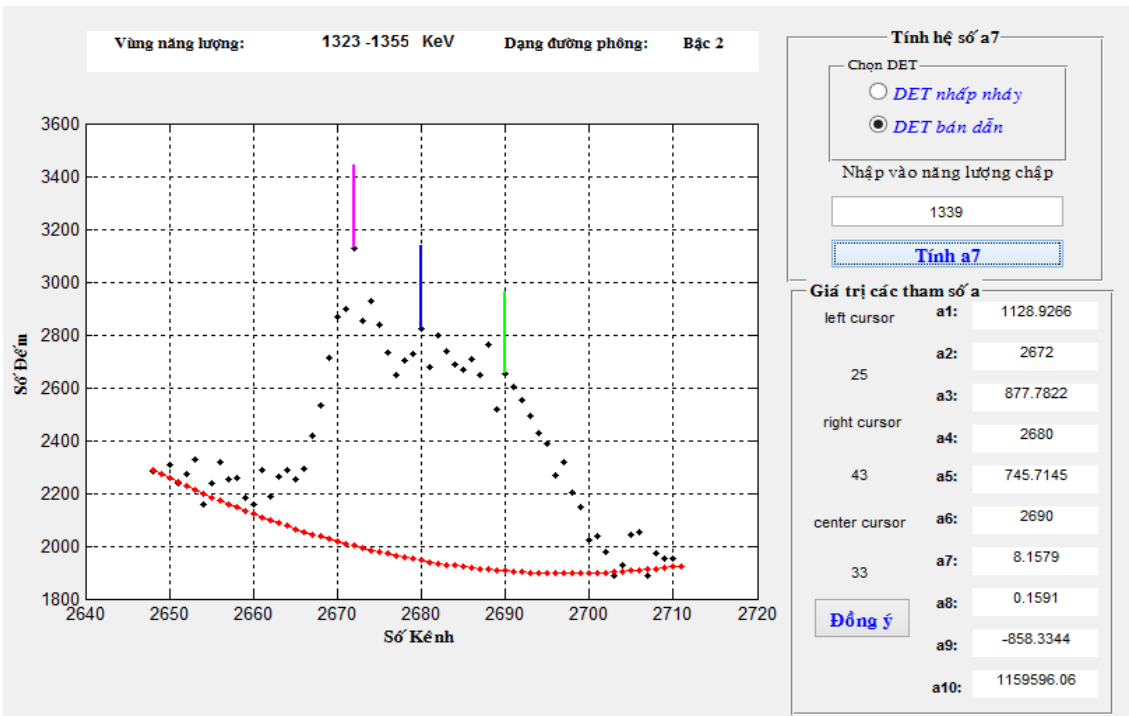

\section{Hình 3. Giao diện xác định các hệ số a từ thực nghiệm}

- Tính các hệ số a bằng thuật toán và tách đỉnh: Các tham số của hàm xấp xỉ từ các số liệu thực nghiệm thu nhận ban đầu, từ đó cung cấp các thông tin về đỉnh chập mà ta quan tâm. Phương pháp sử dụng để tính toán là thuật toán Levenberg-Marquardt (Jorge, 1997).

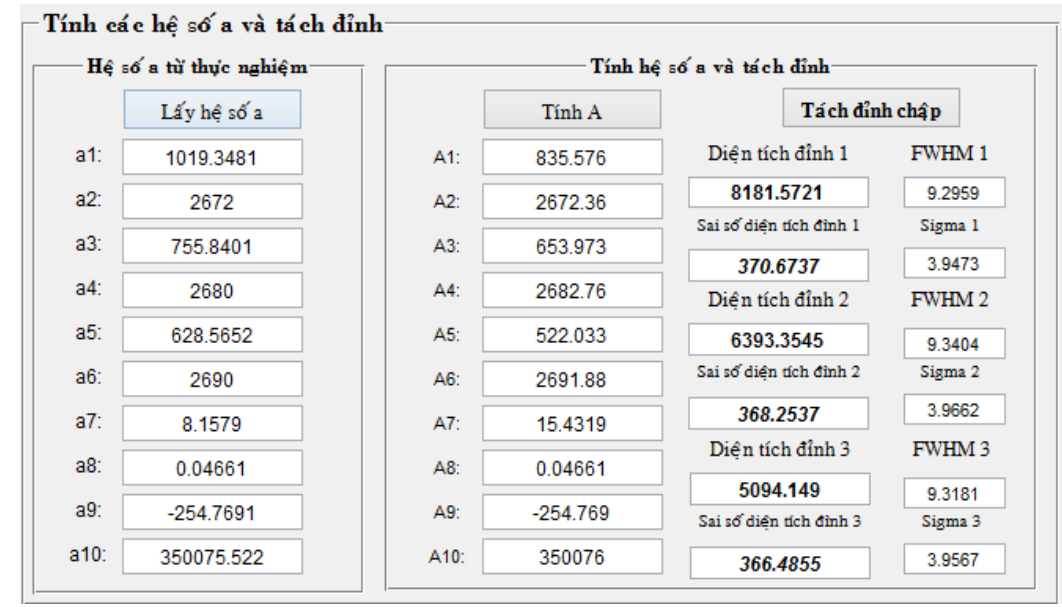

Hình 4. Tính hệ số a bằng thuật toán và tách đỉnh

\section{KẾT QUẢ}

\section{1. Đối với hệ detector nhấp nháy}

Thực hiện tách 3 đỉnh $1274.542 \mathrm{keV}$ của ${ }^{22} \mathrm{Na}-1332.502 \mathrm{keV}$ của ${ }^{60} \mathrm{Co}$ và $1408.022 \mathrm{keV}$ của ${ }^{152}$ Eu trong phổ thực nghiệm đo được ở Phòng Thí nghiệm Vật lý Hạt nhân, Khoa Vật lý, Trường Đại học Đà Lạt. Các giá trị tính toán được nêu ở Bảng 1. 
Bảng 1. Giá trị diện tích của đỉnh chập và các đỉnh con

\begin{tabular}{lcccccccc}
\hline \multirow{2}{*}{ Phông } & \multirow{2}{*}{$\begin{array}{c}\text { Diện tích } \\
\text { đỉnh chập }\end{array}$} & \multirow{2}{*}{$\begin{array}{c}\text { Tổng diện tích } \\
\text { 3 đinh }\end{array}$} & \multicolumn{2}{c}{ Đỉnh 1 } & \multicolumn{2}{c}{ Đỉnh 2 } & \multicolumn{3}{c}{ Đỉnh 3 } \\
\cline { 4 - 9 } & & & $\mathrm{S}_{1}$ & $\Delta \mathrm{S}_{1}$ & $\mathrm{~S}_{2}$ & $\Delta \mathrm{S}_{2}$ & $\mathrm{~S}_{3}$ & $\Delta \mathrm{S}_{3}$ \\
\hline B6 & 328781 & 329638 & 128183 & 598 & 135629 & 604 & 65826 & 543 \\
B7 & 330384 & 331437 & 112166 & 583 & 151176 & 615 & 68095 & 544 \\
Bậc 1 & 265775 & 266726 & 113474 & 637 & 111030 & 635 & 42222 & 578 \\
Bậc 2 & 256518 & 257437 & 110099 & 641 & 108130 & 640 & 39208 & 583 \\
\hline
\end{tabular}

Hình vẽ phổ trước và sau khi tách bằng phần mềm lập trình với các đường phông khác nhau được trình bày trên các kết quả của Hình 5 .

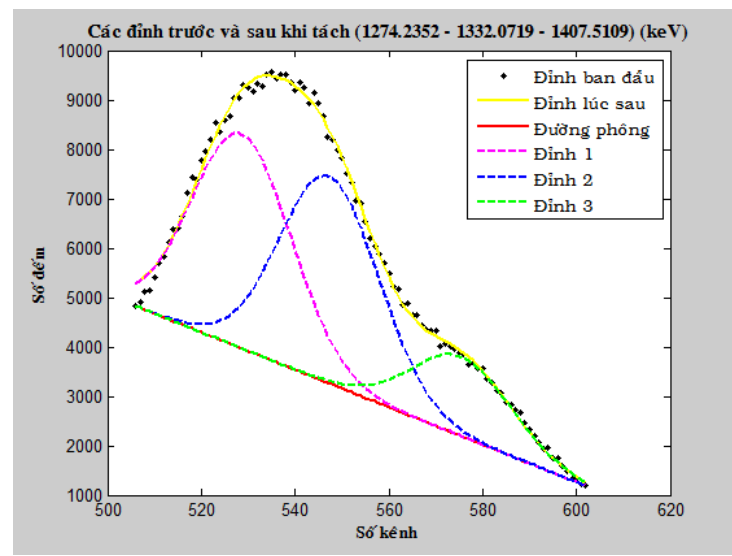

(a)

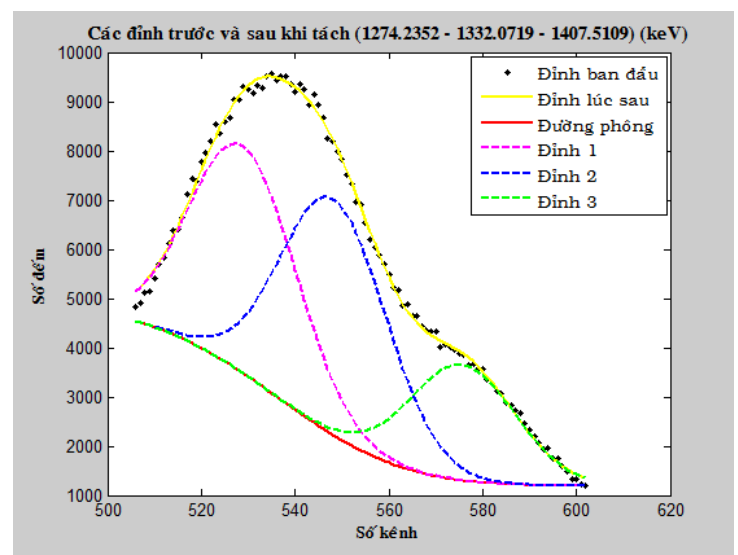

(c)

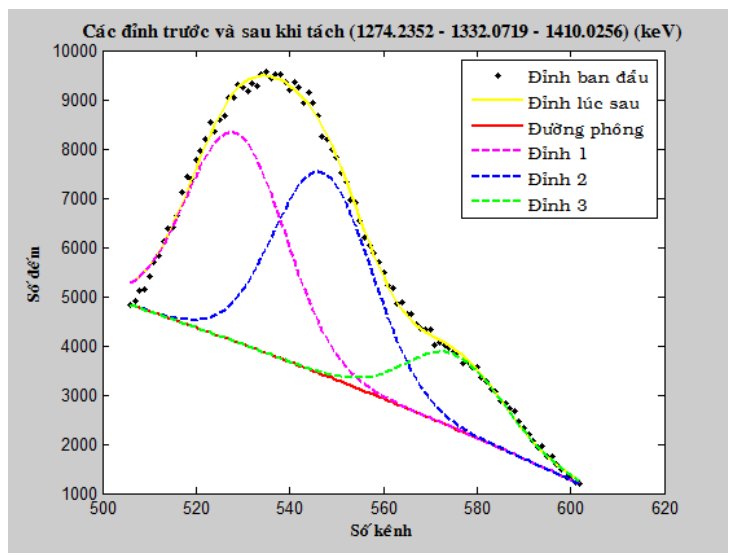

(b)

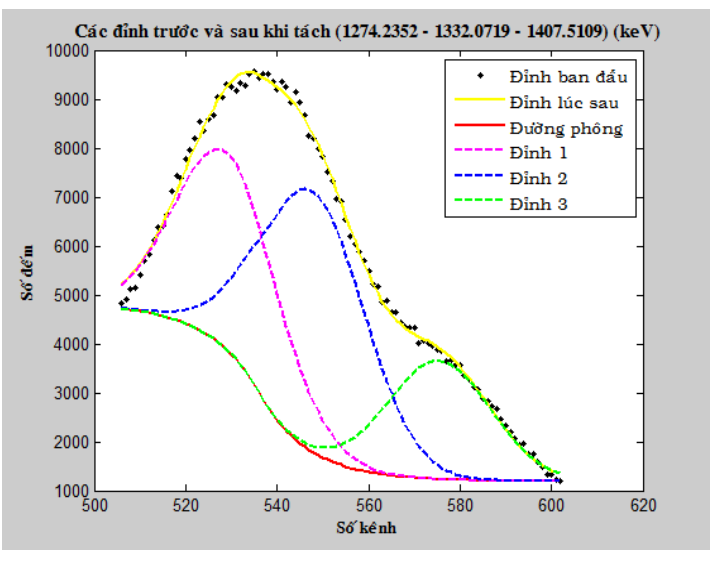

(d)

Hình 5. Đỉnh trước và sau khi tách

Ghi chú: (a) Đường phông bậc 1; (b) Đường phông bậc 2; (c) Đường phông B6; (d) Đường phông $\mathrm{B} 7$

Kết quả tách 3 đỉnh chập $1085.842 \mathrm{keV}\left({ }^{152} \mathrm{Eu}\right)-1112.087 \mathrm{keV}\left({ }^{152} \mathrm{Eu}\right)-$ $1173.238 \mathrm{keV}\left({ }^{60} \mathrm{Co}\right)$ được nêu ở Bảng 2. 
Hình vẽ phổ trước và sau khi tách bằng phần mềm lập trình với các đường phông khác nhau được trình bày bởi kết quả trong Hình 6 .

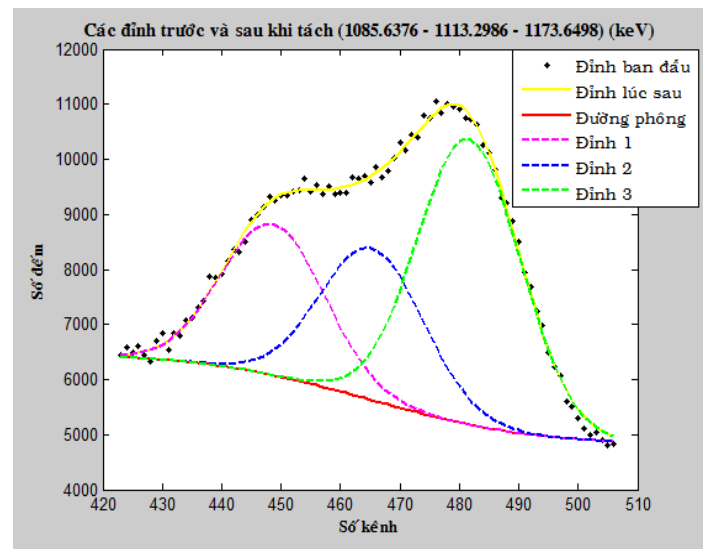

(a)

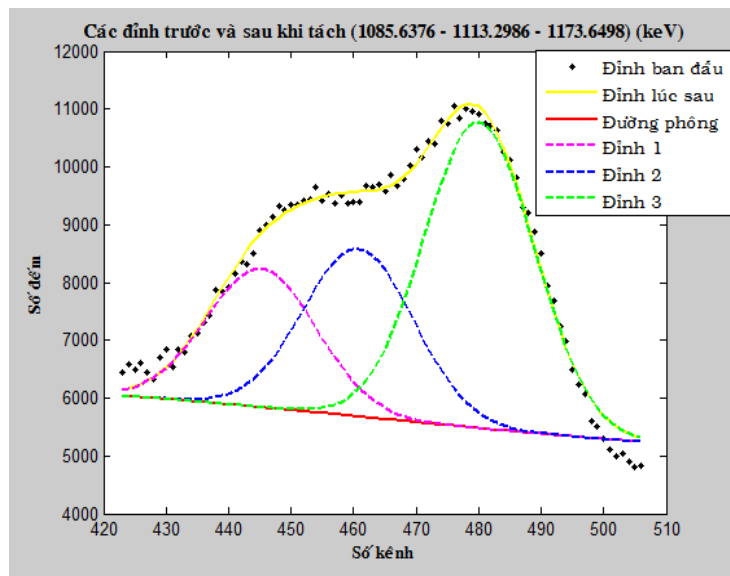

(c)

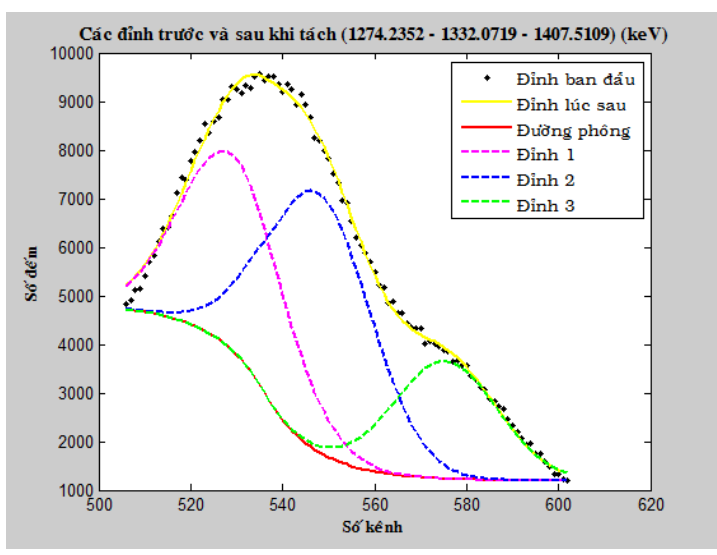

(b)

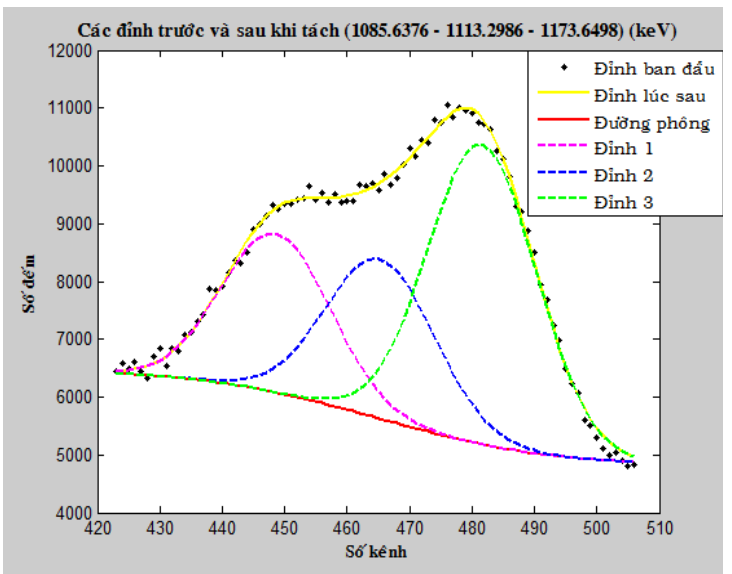

(d)

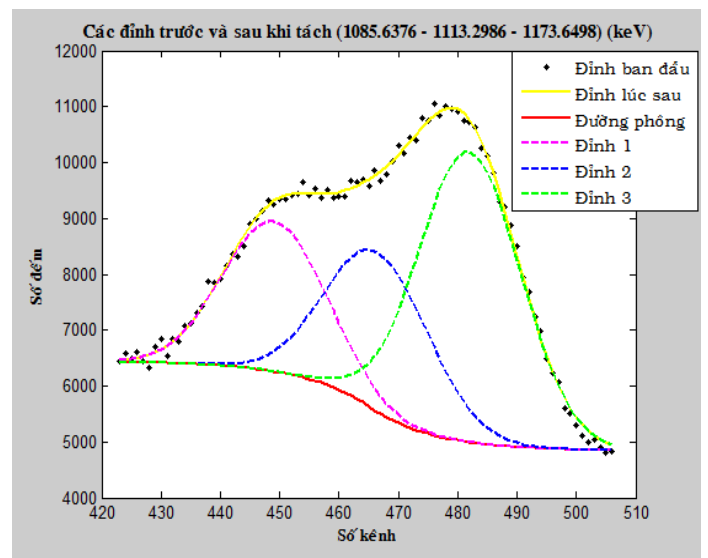

(e)

\section{Hình 6. Đỉnh trước và sau khi tách}

Ghi chú: (a) Đường phông bậc 1; (b) Đường phông bậc 2; (c) Đường phông B5;

(d) Đường phông B6; (e) Đường phông $\mathrm{B} 7$ 
Bảng 2. Giá trị diện tích của đỉnh trước và sau khi tách 3 đỉnh

\begin{tabular}{lccccccccc}
\hline \multirow{2}{*}{ Phông } & \multirow{2}{*}{$\begin{array}{c}\text { Diện tích } \\
\text { đỉnh chập }\end{array}$} & \multirow{2}{*}{$\begin{array}{c}\text { Tổng diện } \\
\text { tích 3 đinh }\end{array}$} & \multicolumn{2}{c}{$\mathrm{S}_{1}$} & $\sigma_{S 1}$ & $\mathrm{~S}_{2}$ & $\sigma_{S 2}$ & $\mathrm{~S}_{3}$ & $\sigma_{S 3}$ \\
\hline B5 & 231916 & 233728 & 52700 & 726 & 64131 & 734 & 116897 & 769 \\
B6 & 231554 & 232365 & 59759 & 731 & 59997 & 731 & 112609 & 766 \\
B7 & 231529 & 232312 & 58229 & 730 & 61756 & 732 & 112327 & 766 \\
Bậc 1 & 233591 & 234327 & 61686 & 731 & 59916 & 729 & 112725 & 765 \\
Bậc 2 & 239203 & 239976 & 63256 & 728 & 61668 & 727 & 115052 & 763 \\
\hline
\end{tabular}

\section{2. Đối với detector bán dẫn}

Tách đỉnh chập ở các phổ phân tích kích hoạt hoạt của mẫu đất đá thu được bằng detector bán dẫn từ Phòng thí nghiệm Phân tích Kích hoạt, Viện Nghiên cứu Hạt nhân Đà Lạt. Tách 3 đỉnh $1335.1 \mathrm{keV}$ - $1340.1 \mathrm{keV}$ - $1344.1 \mathrm{keV}$ với số liệu tách trình bày như trong Bảng 3 . Hình vẽ phổ trước và sau khi tách bằng phần mềm lập trình với các đường phông khác nhau như các kết quả trong Hình 7.

Bảng 3. Giá trị diện tích đỉnh trước và sau khi tách 3 đỉnh

\begin{tabular}{|c|c|c|c|c|c|c|c|c|}
\hline \multirow{2}{*}{ Phông } & \multirow{2}{*}{$\begin{array}{l}\text { Diện tích đỉnh } \\
\text { chập }\end{array}$} & \multirow{2}{*}{$\begin{array}{l}\text { Tổng diện } \\
\text { tích } 3 \text { đỉnh }\end{array}$} & \multicolumn{2}{|c|}{ Đỉnh 1} & \multicolumn{2}{|c|}{ Đỉnh 2} & \multicolumn{2}{|c|}{ Đỉnh 3} \\
\hline & & & $\mathrm{S}_{1}$ & $\sigma_{S_{1}}$ & $\mathrm{~S}_{2}$ & $\sigma_{S_{2}}$ & $\mathrm{~S}_{3}$ & $\sigma_{S_{3}}$ \\
\hline B5 & 18227 & 18035 & 7558 & 372 & 5793 & 370 & 4684 & 369 \\
\hline B6 & 20199 & 20103 & 8067 & 339 & 6629 & 336 & 5407 & 335 \\
\hline B7 & 20206 & 19999 & 7715 & 338 & 6824 & 337 & 5460 & 335 \\
\hline Bậc 1 & 18510 & 18403 & 7652 & 372 & 5946 & 370 & 4805 & 368 \\
\hline Bậc 2 & 20102 & 19669 & 8182 & 371 & 6393 & 368 & 5094 & 367 \\
\hline
\end{tabular}

Kết quả cho thấy, chương trình tính toán và tách các đỉnh chập trong phổ các Gamma đo bằng detector nhấp nháy và detector bán dẫn cho kết quả diện tích tổng cộng của các đỉnh con tương đương với diện tổng của đỉnh chập, tuy nhiên vẫn có sự sai khác trong các trường hợp tách là do có sự thăng giáng trong thống kê và việc lựa chọn đường phông để tách các đỉnh chập. Như vậy, kết quả cho thấy phần mềm tự thiết kế đáp ứng được yêu cầu của bài toán tách ba đỉnh chập trong xử lý, phân tích phổ năng lượng Gamma. 


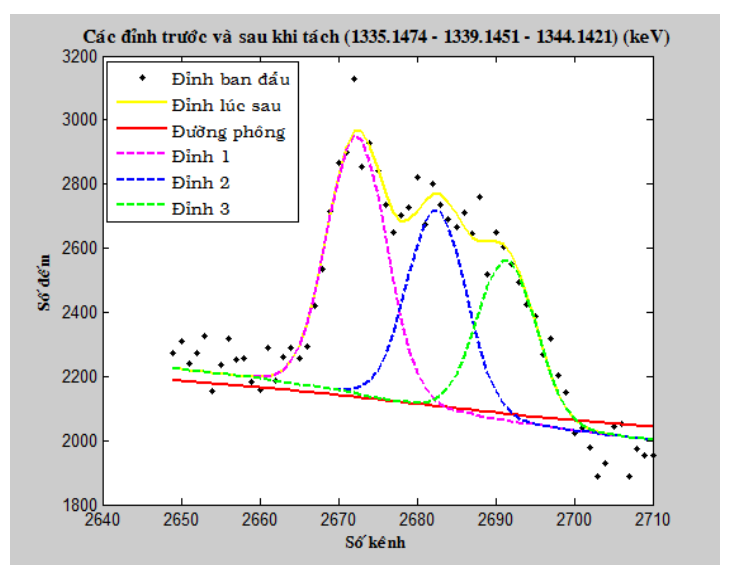

(a)

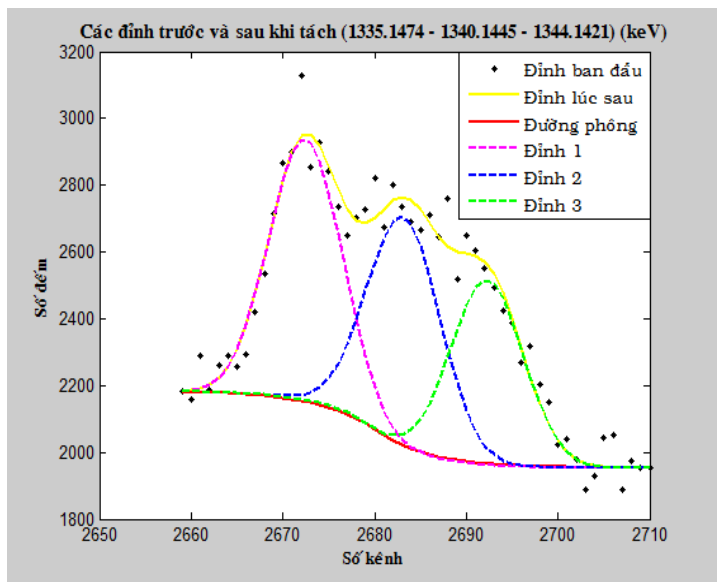

(c)

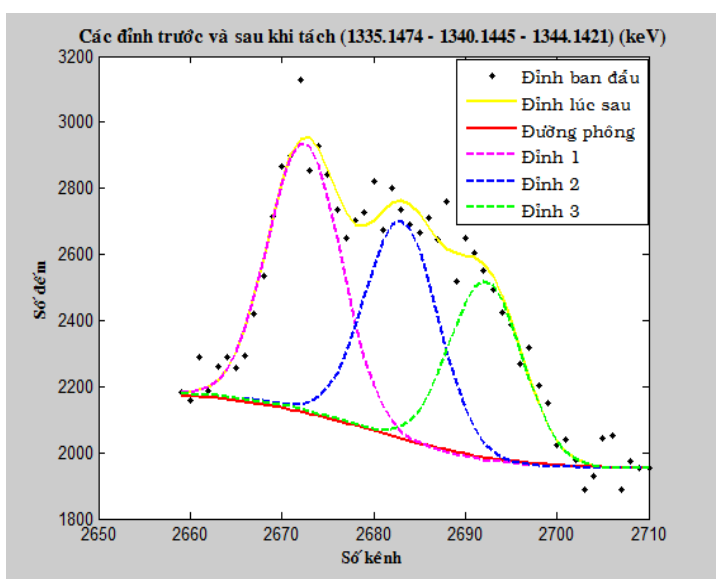

(b)

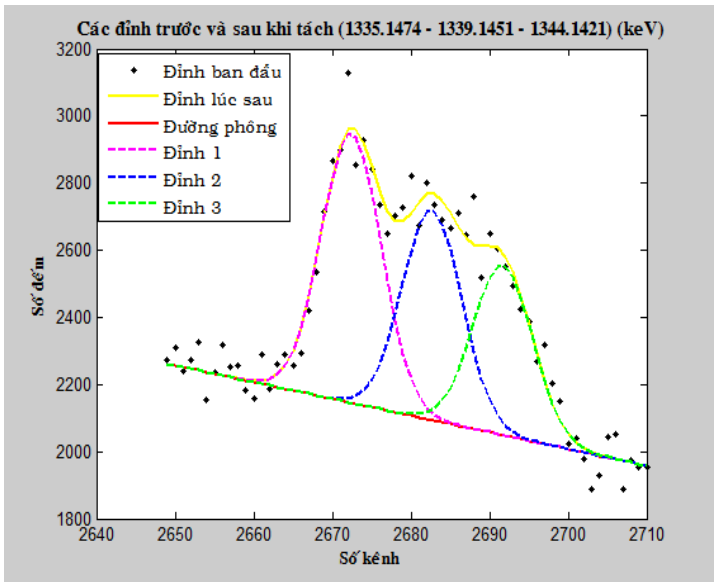

(d)

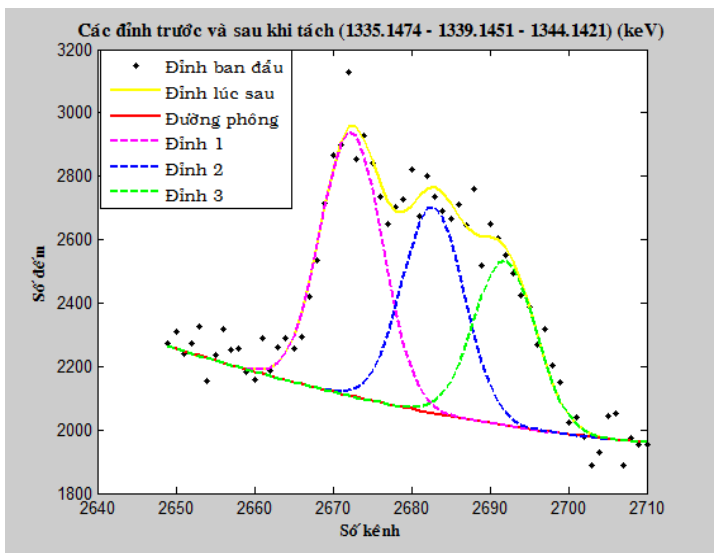

(e)

Hình 7. Đỉnh trước và sau khi tách

Ghi chú: (a) Đường phông B5; (b) Đường phông B6; (c) Đường phông B7;

(d) Đường phông Bậc 1; (e) Đường phông Bậc 2 


\section{KẾT LUẬn}

Bằng thuật toán Levenberg-Marquardt, với việc tách ba đỉnh chập trên nền phông tuyến tính và phi tuyến, nhóm nghiên cứu đã xây dựng chương trình trên ngôn ngữ lập trình Matlab. Kết quả bước đầu đã xử lý, tách các đỉnh chập trong dải năng lượng rộng đối với detector nhấp nháy và các đỉnh chập trong dải năng lượng hẹp đối với detector bán dẫn. Chương trình đã xây dựng là một công cụ tính toán rất thích hợp trong các phòng thí nghiệm phân tích phổ bức xạ hoặc có thể phối hợp với các phần mềm thương mại khác trong việc tách các đỉnh chập.

\section{TÀI LIỆU THAM KHẢO}

David, G. L., \& Yinyu, Y. (2008). Linear and nonlinear programming. Berlin, Gemany: Springer.

John, F. M. (2001). Numerical methods of statistics. New York, USA: Cambrige University Press.

John, N., Michael, H. K., Christopher, J. N., \& William, W. (1990). Applied linear statistical models. New York, USA: McGraw-Hill Press.

Jorge, J. M. (1997). The Levenberg-Marquardt algorithm: Implementation and theory. Berlin, Gemany: Springer.

Mai, X. T., \& Võ, T. A. Q. (2015). Xây dựng phần mềm tách các đỉnh chập của phổ Gamma với các phông phi tuyến và tuyến tính. Tạp chí Khoa học Đại học Đà Lạt, $5,7-17$.

Nguyễn, T. H., \& Nguyễn, V. A. (2006). Lập trình Matlab và ứng dụng. Hà nội, Việt Nam: NXB Khoa học và Kỹ thuật.

Phạm, T. N. Y., Ngô, H. T., Lê, T. H., \& Nguyễn, T. L. H. (2007). Co sở Matlab và úng dụng. Hà Nội, Việt Nam: NXB Khoa học và Kỹ thuật. 


\title{
THE TECHNIQUES SEPARATING THREE OVERLAP PEAKS IN GAMMA SPECTRUM
}

\author{
Trinh Ngoc Phap ${ }^{a^{*},}$ Mai Xuan Trung ${ }^{b}$
}

${ }^{a}$ The Library, Dalat University, Lamdong, Vietnam

${ }^{b}$ The Faculty of Nuclear Engineering, Dalat University, Lamdong, Vietnam

*Corresponding author: Email: phaptn@dlu.edu.vn

\section{Article history}

Received: October $08^{\text {th }}, 2015$ | Received in revised form: April 20 ${ }^{\text {th }}, 2016$

Accepted: August $29^{\text {th }}, 2016$

\begin{abstract}
Separating the three overlapped peaks of a Gamma spectrum is essential requirements in experimental nuclear physics as well as in analysis of gamma-ray spectrum. In this paper, we present the results of calculations for the problem of overlap peaks, in which three individual peaks are overlapped with each other. The calculation procedure was performed by the Levenberg-Marquardt algorithm with linear and nonlinear background. Based on separating the two overlap peaks method has been implemented in our previous article, the separating three overlap peaks was successfully implemented in this paper, in which the calculations were tested with gamma-ray spectra by using a Semiconductor detector and a Scintillator detector.
\end{abstract}

Keywords: Gamma spectrum; Levenberg-Marquardt algorithm. Overlapping peaks separation; 